\title{
One-pot synthesis of novel $\alpha$-amino phosphonates using tetramethylguanidine as a catalyst
}

\author{
M. V. Narayana Reddy, ${ }^{a}$ B. Siva Kumar, ${ }^{a}$ A. Balakrishna, ${ }^{a}$ C. S. Reddy, ${ }^{* a}$ S. K. Nayak, \\ and C. D. Reddy ${ }^{a}$ \\ ${ }^{a}$ Department of Chemistry, Sri Venkateswara University, Tirupati-517 502, India \\ ${ }^{b}$ Bio-Organic Division, BARC, Mumbai-400085 \\ E-mail: csureshsvu@yahoo.com
}

\begin{abstract}
$\alpha$-Aminophosphonates (4a-l) were synthesized in high yields by the Kabachnik-Fields reaction. One-pot simultaneous reaction of indole-3-carboxaldehyde, a dialkyl- or diphenyl phosphite, and different heterocyclic-, cyclic-, or other primary amines in the presence of tetramethylguanidine (10 mole \%) as catalyst in toluene at reflux temperature afforded 4a-l. They exhibited moderate to high antimicrobial activity.
\end{abstract}

Keywords: $\alpha$-Aminophosphonate, antimicrobial activity, indole-3-carboxaldehyde

\section{Introduction}

The synthesis of $\alpha$-aminophosphonates exhibiting high bio-activity has recently attracted a lot of attention. ${ }^{1-3}$ Their diverse applications include inhibition of synthase, ${ }^{4}$ HIV protease, ${ }^{5}$ renin, ${ }^{6}$ and PTPases; ${ }^{7,8}$ some of these derivatives are potential antibiotics ${ }^{9}$ or herbicides. ${ }^{10} \alpha$-Aminophosphonates are chief substrates in the synthesis of phosphonopeptides. ${ }^{11}$ Due to their structural analogy with $\alpha$-amino acids, these types of organophosphorus compounds are widely used for the development of new inhibitors of enzymes, neuroactive compounds, and plant growth regulators. ${ }^{12,13}$ Among the number of synthetic approaches to $\alpha$-aminophosphonates, one of the most powerful methods is the Kabachnik-Fields reaction. ${ }^{14,15}$ Previous results demonstrated that tetramethylguanidine (TMG) catalyzes the Michael addition of nitromethane to $\alpha, \beta$-unsaturated ketones. ${ }^{16,17}$ TMG has been used only sporadically and has not yet received full recognition as a strong base in organic synthesis. Its catalytic activity in the Kabachnik-Fields reaction is explored in the present investigation. 


\section{Results and Discussion}

Indole 3-carboxaldehyde (1) was treated with 2-aminobenzothiazole (3a), 3-amino-5methylisoxazole (3d), phenylglycine ethyl ester $(\mathbf{3 g})$, or cyclohexylamine $(\mathbf{3} \mathbf{j})$, and dimethyl-, diethyl-, or diphenyl phosphite in the presence of 10 mole \% of tetramethylguanidine (TMG) in dry toluene at RT. The mixture was stirred at RT for $1 \mathrm{~h}$, and at $70-80^{\circ} \mathrm{C}$ for another $5 \mathrm{~h}$. The progress of the reaction was monitored by thin layer chromatography. The reaction proceeded smoothly, and completed in 5-6h to afford the corresponding $\alpha$-aminophosphonates in high yield (60-85\%). This showed that TMG acts as an effective catalyst in this reaction. An important feature is that the TMG can be easily recovered from the reaction mixture after completion of the reaction and can be reused. The chemical structures of all the new compounds were confirmed by elemental analysis, IR, ${ }^{1} \mathrm{H}-,{ }^{13} \mathrm{C}$ - and ${ }^{31} \mathrm{P}$ - NMR spectra. Compounds 4a-l exhibited characteristic IR stretching frequencies in the regions $3220-3390,1208-1238,746-780 \mathrm{~cm}^{-1}$ for $\mathrm{N}-\mathrm{H}, \mathrm{P}=\mathrm{O}$, and $\mathrm{P}-\mathrm{C}\left(\right.$ aliphatic) respectively. ${ }^{18}$

The aromatic protons of the benzene rings of the $\alpha$-amino-phosphonates (4a-l) showed a complex multiplet at $\delta 6.81-7.96$. The P-C-H proton signal appeared as a multiplet ${ }^{19 a}$ at $\delta 4.74-$ 4.89 due to its coupling with both phosphorus and the N-H proton. The exocyclic N-H proton signal appeared at $\delta 4.90-5.90$ as a singlet. The indole N-H proton resonated at $\delta 9.89-10.18$ as a singlet. The methylene protons of P-O-C $\underline{H}_{2}-\mathrm{CH}_{3}$ showed a multiplet, and methyl protons of P-O$\mathrm{CH}_{2}-\underline{\mathrm{C}}_{3}$ gave triplets in the region $\delta 3.75-3.96$ and $1.18-1.78$ respectively. ${ }^{19 \mathrm{~b}}$ The methoxy group protons of the dimethylphosphite moiety resonated as two distinct doublets in the range of $\delta 3.14-3.61\left(\mathrm{~d},{ }^{3} \mathrm{~J}_{\mathrm{P}-\mathrm{H}}=10.0-11.4 \mathrm{~Hz}\right)$ and $\delta 3.51-3.70\left(\mathrm{~d},{ }^{3} \mathrm{~J}_{\mathrm{P}-\mathrm{H}}=9.80-11.30\right){ }^{19,20}$ indicating their non-equivalence.

The carbon chemical shifts for P- $\underline{\mathrm{CH}}-, \mathrm{P}-\mathrm{O}-\underline{\mathrm{C}} \mathrm{H}_{3}$ and $\mathrm{P}-\mathrm{O}-\underline{\mathrm{C}} \mathrm{H}_{2}-\underline{\mathrm{C}} \mathrm{H}_{3}$ in the title compounds were observed at $\delta 54.0,55.6,62.8,16.2$ respectively. ${ }^{18,21,22}$ The $^{31} \mathrm{P}$ NMR signals ${ }^{23}$ appeared in the region $\delta 20.30-31.80$ for these compounds.

\section{Experimental Section}

General Procedures. IR spectra were recorded in $\mathrm{KBr}$ pellets on a Perkin-Elmer 683 spectrophotometer. ${ }^{1} \mathrm{H}$ - NMR spectra were recorded at $300 \mathrm{MHz}$ in $\mathrm{CDCl}_{3}$ using TMS as internal standard reference. ${ }^{31} \mathrm{P}$ - NMR $(121.4 \mathrm{MHz})$ was taken in $\mathrm{CDCl}_{3}$ using $85 \% \mathrm{H}_{3} \mathrm{PO}_{4}$ as external standard with broadband ${ }^{1} \mathrm{H}$ - decoupling. ${ }^{13} \mathrm{C}$ - NMR spectra measurements were performed at 75.4 MHz using TMS as internal standard. ${ }^{1} \mathrm{H}-,{ }^{13} \mathrm{C}-,{ }^{31} \mathrm{P}-\mathrm{NMR}$ spectra were taken on Varian Gemini $300 \mathrm{MHz}$ spectrometer and mass spectra were recorded on JEOL MSD- instrument. RT denotes room temperature. 


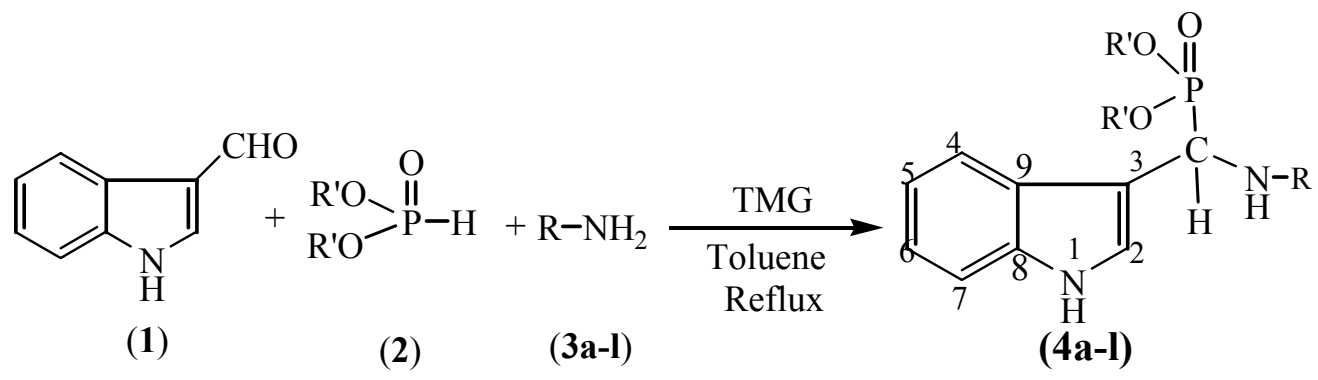

\begin{tabular}{|c|c|c|}
\hline Compound & $\mathrm{R}^{\prime}$ & $\mathrm{R}$ \\
\hline $4 a$ & $\mathrm{CH}_{3}$ & \\
\hline $4 b$ & $\mathrm{C}_{2} \mathrm{H}_{5}$ & , \\
\hline $4 c$ & $\mathrm{C}_{6} \mathrm{H}_{5}$ & " \\
\hline $4 d$ & $\mathrm{CH}_{3}$ & \\
\hline $4 e$ & $\mathrm{C}_{2} \mathrm{H}_{5}$ & , \\
\hline $4 f$ & $\mathrm{C}_{6} \mathrm{H}_{5}$ & , \\
\hline $4 g$ & $\mathrm{CH}_{3}$ & \\
\hline $4 h$ & $\mathrm{C}_{2} \mathrm{H}_{5}$ & , \\
\hline $4 i$ & $\mathrm{C}_{6} \mathrm{H}_{5}$ & \\
\hline $4 j$ & $\mathrm{CH}_{3}$ & \\
\hline $4 k$ & $\mathrm{C}_{2} \mathrm{H}_{5}$ & " \\
\hline 41 & $\mathrm{C}_{6} \mathrm{H}_{5}$ & 证 \\
\hline
\end{tabular}

Scheme 1

\section{Antimicrobial activity}

The antibacterial activity of (4a-I) was assayed ${ }^{24}$ against the growth of Staphylococcus aureus (gram +ve) and Escherichia coli (gram -ve) at three concentrations (100, 50, 25 ppm) (Table 1). The majority of the compounds exhibited high activity against both the bacteria. The highlight is that the two compounds, diphenyl (benzo[d]thiazol-2-ylamino)(1H-indol-3-yl)methyl phosphonate (4c) and dimethyl (5-methylisoxazol-3-ylamino)(1H-indol-3-yl)methyl phosphonate (4d) were more effective than the standard penicillin. 


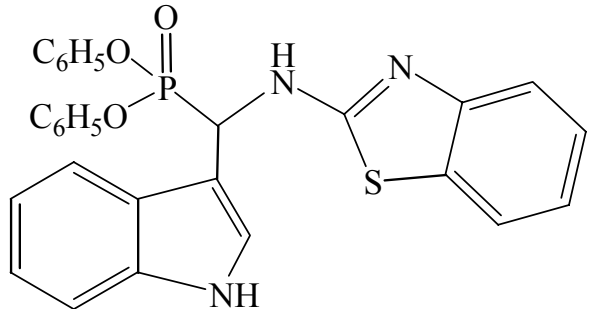

(4c)

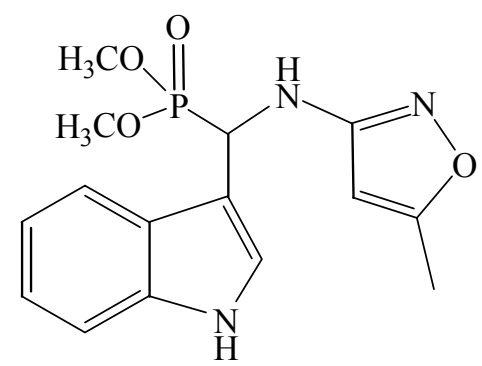

(4d)<smiles>CCOC(=O)C(NC(c1ccccc1)P(=O)(O)OC)c1c[nH]c2ccccc12</smiles>

(4g)

Table 1. Antibacterial activity of $\alpha$-aminophosphonates (4a-l)

\begin{tabular}{|c|c|c|c|c|c|c|}
\hline \multirow[t]{3}{*}{ Compound } & \multicolumn{6}{|c|}{ Zone of inhibition (\%) } \\
\hline & \multicolumn{3}{|c|}{ Escherichia coli } & \multicolumn{3}{|c|}{ Staphylococcus aureus } \\
\hline & 100 & 50 & 25 & 100 & 50 & 25 \\
\hline $4 a$ & 13 & 9 & 5 & 10 & 8 & 6 \\
\hline $4 b$ & 12 & 7 & 4 & 11 & 8 & 6 \\
\hline $4 c$ & 13 & 11 & 7 & 12 & 9 & 5 \\
\hline $4 d$ & 15 & 12 & 5 & 16 & 10 & 8 \\
\hline $4 e$ & 11 & 7 & 8 & 10 & 8 & 5 \\
\hline $4 f$ & 10 & 6 & 4 & 15 & 11 & 8 \\
\hline $4 \mathrm{~g}$ & 12 & 5 & 5 & 10 & 8 & 5 \\
\hline $4 h$ & 7 & 8 & 6 & - & - & - \\
\hline $4 \mathbf{i}$ & 9 & 7 & 6 & 10 & 7 & 5 \\
\hline $4 \mathbf{j}$ & 10 & 5 & 5 & 9 & 7 & 6 \\
\hline $4 \mathbf{k}$ & 12 & 9 & 6 & 10 & 8 & 6 \\
\hline 41 & 10 & 9 & 6 & 9 & 8 & 6 \\
\hline Penicillin & 12 & 8 & - & 10 & 7 & - \\
\hline
\end{tabular}


The compounds 4a-l were screened for their antifungal activity against Aspergillus niger and Helminthosporium oryzae species along with the standard fungicide Griseofulvin (Table 2) by the disc diffusion method ${ }^{25}$ at three different concentrations $(100,50,25 \mathrm{ppm})$.

It is gratifying to observe that the majority of the compounds 4a-I exhibited higher antifungal activity when compared with the Griseofulvin reference. Significant is the fact that diphenylbenzo[ $d]$ thiazol-2-ylamino-1H-indol-3-ylmethyl phosphonate (4c), and dimethylethoxycarbonyl(phenyl)methylamino)(1H-indol-3-yl) methyl phosphonate (4g) exhibited higher activity than the standard Griseofulvin against both the fungi. Thus new compounds with very high antimicrobial/fungicidal activity than the presently used commercial bactericides / fungicides have been discovered.

Table 2. Antifungal activity of $\alpha$-aminophosphonates (4a-l)

\begin{tabular}{ccccccc}
\hline \multirow{2}{*}{ Compound } & \multicolumn{6}{c}{ Zone of inhibition (\%) } \\
\cline { 2 - 7 } & \multicolumn{7}{c}{ Aspergillus Niger } & \multicolumn{4}{c}{ Helminthosporium oryzae } \\
\cline { 2 - 7 } & 100 & 50 & 25 & 100 & 50 & 25 \\
\hline $\mathbf{4 a}$ & 11 & 6 & 4 & 11 & 6 & 5 \\
$\mathbf{4 b}$ & 12 & 9 & 5 & 11 & 9 & 5 \\
$\mathbf{4 c}$ & 14 & 8 & 8 & 13 & 10 & 7 \\
$\mathbf{4 d}$ & 11 & 10 & 6 & 15 & 9 & 4 \\
$\mathbf{4 e}$ & 10 & 5 & 4 & 13 & 11 & 9 \\
$\mathbf{4 f}$ & 9 & 7 & 9 & 9 & 8 & - \\
$\mathbf{4 g}$ & 12 & 11 & 8 & 13 & 12 & 8 \\
$\mathbf{4 h}$ & 14 & 8 & 9 & 10 & 9 & 7 \\
$\mathbf{4 i}$ & 11 & 10 & 9 & 11 & 9 & 5 \\
$\mathbf{4 j}$ & 10 & 8 & 7 & 12 & 7 & 8 \\
$\mathbf{4 k}$ & 12 & 10 & 8 & 14 & 10 & 7 \\
$\mathbf{4 l}$ & 10 & 8 & 7 & 12 & 10 & 7 \\
Griseofulvin & 10 & 7 & - & 12 & 9 & - \\
\hline
\end{tabular}

\section{General procedure for the synthesis of $\alpha$ - aminophosphonates (4a-l)}

To a stirred solution of indol-3-carboxaldehyde (1) $(0.0725 \mathrm{~g}, 0.005$ mole) the amine (3a-l) $(0.005$ mole $)$ in anhydrous toluene $(20 \mathrm{~mL})$ was added dropwise, and then TMG (10 mole \%) was added and stirring continued at RT for $1 \mathrm{~h}$. Then dimethyl-, diethyl-, or diphenyl phosphite (2) $(0.72 \mathrm{~g}, 0.005$ mole) in anhydrous toluene $(20 \mathrm{~mL})$ was added dropwise. Stirring was continued at RT for another $0.5 \mathrm{~h}$, then the mixture was heated at gentle reflux for 5-6h. The progress of the reaction was monitored by TLC analysis. After completion of the reaction the solvent was removed under reduced pressure. The residue was purified by column chromatography on silica gel (80-120 mesh) using petroleum-ethyl acetate (8:3) as eluent. 
Dimethyl (benzo $[\boldsymbol{d}]$ thiazol-2-ylamino)(1H-indol-3-yl)methyl phosphonate (4a). Yield 85\% mp, 105-106 ${ }^{\circ}$ C. IR (KBr) cm $3250(\mathrm{P}-\mathrm{N}-\mathrm{H}), 1218(\mathrm{P}=\mathrm{O}), 750(\mathrm{P}-\mathrm{CH}) ;{ }^{1} \mathrm{H}-\mathrm{NMR}$ (DMSO-d 6 ): $\delta$ 6.87-7.63 (m, 9H, Ar-H), 9.98 (s, 1H, Ar-NH), 5.91 (s, 1H, Aliph-NH), 4.74-4.84 (m, 1H, P$\mathrm{CH}), 3.37-3.42\left(\mathrm{~d},{ }^{3} J_{\mathrm{P}-\mathrm{H}}=10.2 \mathrm{~Hz}, \mathrm{P}-\mathrm{OCH}_{3}\right), 3.53-3.63\left(\mathrm{~d},{ }^{3} \mathrm{~J}_{\mathrm{P}-\mathrm{H}}=10.8 \mathrm{~Hz}, \mathrm{P}-\mathrm{OCH}_{3}\right) ;{ }^{13} \mathrm{C}-\mathrm{NMR}$ (DMSO-d 6 ), $\delta 122.8$ (C-2), 112.1 (C-3), 120.5 (C-4), 121.7 (C-5), 119.6 (C-6), 111.0 (C-7), 131.6 (C-8), 136.5 (C-9), 179 (C-2'), 122.1 (C-4'), 125.8 (C-5'), 122.7 (C-6'), 148.9 (C-7'), 148.9 (C-8'), 125.0 (C-9'), 54.4(P-CH), $55.0\left(\mathrm{OCH}_{3}, \mathrm{~d}, J=6.5 \mathrm{~Hz}\right) ;{ }^{31} \mathrm{P}-\mathrm{NMR}$ (DMSO-d 6 ): $\delta$ 28.27; GCMS m/z (\%): $388\left(\mathrm{M}^{+} 6\right), 238.0$ (8), 165.1 (100), 151.1 (5), 150.0 (40), 123.1 (4); Anal. Calcd. for $\mathrm{C}_{18} \mathrm{H}_{18} \mathrm{~N}_{3} \mathrm{O}_{3}$ PS: C, 55.80; H, 4.68; N, 10.84. Found: C, 55.60; H, 4.58; N, 10.70\%.

Diethyl (benzo[d]thiazol-2-ylamino)(1H-indol-3-yl)methyl phosphonate (4b). Yield (80\%) mp 109-110 ${ }^{\circ}$; IR (KBr) cm ${ }^{-1} ; 3380(\mathrm{P}-\mathrm{N}-\mathrm{H}), 1216(\mathrm{P}=\mathrm{O}), 759(\mathrm{P}-\mathrm{CH}) ;{ }^{1} \mathrm{H}-\mathrm{NMR}\left(\mathrm{DMSO}-\mathrm{d}_{6}\right) \delta$ 6.83-7.63 (m, 9H, Ar-H), 9.98 (s, 1H, Ar-NH), 5.91 (s, 1H, Aliph-NH), 4.74-4.81 (m, 1H,P-CH), 3.69-3.81 (m, 4H, P-O-C $\underline{H}_{2} 2$, 1.29-1.80 (m, 6H, P-O-CH $\left.{ }_{2}-\underline{C H}_{3}\right) ;{ }^{13} \mathrm{C}$ NMR $\left(\mathrm{DMSO}-\mathrm{d}_{6}\right) \delta 123.0$ (C-2, 110.1 (C-3), 122.8 (C-4), 122.9 (C-5), 120.2 (C-6), 113.0 (C-7), 130.8 (C-8), 137.0 (C-9), 178 (C-2'), 121.8 (C-4'), 126.8 (C-5'), 122.9 (C-6'), 147.9 (C-7'), 146.8 (C-8'), 123.0 (C-9'), 55.1 $(\mathrm{P}-\mathrm{CH}), 62.8\left(\mathrm{O}-\underline{\mathrm{C}}_{2}-\mathrm{CH}_{3}, \mathrm{~d}, J=6.9 \mathrm{~Hz}\right), 16.2\left(\mathrm{OCH}_{2}-\underline{\mathrm{C}}_{3}, \mathrm{~d}, J=12.0 \mathrm{~Hz}\right) ;{ }^{31} \mathrm{P}$ NMR $\left(\mathrm{DMSO}-\mathrm{d}_{6}\right)$; $\delta$ 26.20. Anal. Calcd. for $\mathrm{C}_{20} \mathrm{H}_{22} \mathrm{~N}_{3} \mathrm{O}_{3} \mathrm{PSC}, 58.10 ; \mathrm{H}, 4.87 ; \mathrm{N}, 10.16$. Found: C, 58.00, H, 4.80; N, $10.14 \%$.

Diphenyl (benzo $[\boldsymbol{d}]$ thiazol-2-ylamino)(1H-indol-3-yl)methyl phosphonate (4c). Yield 85\% mp 80-81 ${ }^{\circ} \mathrm{C}$; IR (KBr) cm ${ }^{-1} ; 3345(\mathrm{P}-\mathrm{NH}), 1215(\mathrm{P}=\mathrm{O}), 770(\mathrm{P}-\mathrm{CH}) ;{ }^{1} \mathrm{H}-\mathrm{NMR}$ (DMSO- $\left.d_{6}\right) \delta$ 6.76-7.71 (m, 19H, Ar-H), 9.96 (s, 1H, Ar-NH), 5.90 (s, 1H, Aliph-NH), 4.71-4.82 (m, 1H,P$\mathrm{CH}) ;{ }^{31} \mathrm{P}$ NMR (DMSO-d $d_{6}$ ); $\delta$ 23.58. Anal. Calcd. for $\mathrm{C}_{28} \mathrm{H}_{22} \mathrm{~N}_{3} \mathrm{O}_{3} \mathrm{PS}: \mathrm{C}, 66.00 ; \mathrm{H}, 3.95 ; \mathrm{N}, 8.24$. Found: C, 65.90; H, 3.92; N, 8.19\%.

Dimethyl (5-methylisoxazol-3-ylamino)(1H-indol-3-yl)methyl phosphonates (4d). Yield 75\% mp 75-76 C; IR (KBr) cm ${ }^{-1} ; 3390(\mathrm{P}-\mathrm{NH}), 1225$ (P=O), $746(\mathrm{P}-\mathrm{CH}) ;{ }^{1} \mathrm{H}-\mathrm{NMR}$ (DMSO- $\left.d_{6}\right) \delta$ 6.84-7.70 (m, 6H, Ar-H), 10.12 (s, 1H, Ar-NH), 5.70 (s, 1H, Aliph-NH), 4.76-4.88 (m, 1H, P$\mathrm{CH}) ; 3.28-3.35\left(\mathrm{~d},{ }^{3} J_{\mathrm{P}-\mathrm{H}}=10.0 \mathrm{~Hz}, \mathrm{P}-\mathrm{OCH}_{3}\right), 3.57-3.68\left(\mathrm{~d},{ }^{3} J_{\mathrm{P}-\mathrm{H}}=11.1 \mathrm{~Hz}, \mathrm{P}-\mathrm{OCH}_{3}\right) 2.10(\mathrm{~s}, 3 \mathrm{H}$, Ar- $\left.\mathrm{CH}_{3}\right) ;{ }^{13} \mathrm{C}$ NMR (DMSO- $\left.d_{6}\right) \delta 122.7$ (C-2), 112.5 (C-3), 121.8 (C-4), 120.6 (C-5), $119.6(\mathrm{C}-$ 6), 111.5 (C-7), 131.2 (C-8), 179 (C-9), 151.5 (C-3'),113.5(C-4'), 160.9 (C-5'),54.76 (P-CH), $55.0\left(\mathrm{P}-\mathrm{OCH}_{3}, \mathrm{~d}, J=6.5 \mathrm{~Hz}\right), 15.8\left(\mathrm{Ar}-\mathrm{CH}_{3}\right) ;{ }^{31} \mathrm{P}$ NMR (DMSO- $\left.d_{6}\right) ; \delta 31.29 ; \mathrm{GC}-\mathrm{MS} \mathrm{m} / \mathrm{z}(\%)$ : 335.8 ( $\left.\mathrm{M}^{+} 20\right), 245$ (35), 237.9 (100), 226.0 (50), 142.9 (30); Anal. Calcd. for $\mathrm{C}_{15} \mathrm{H}_{18} \mathrm{~N}_{3} \mathrm{O}_{4} \mathrm{P}: \mathrm{C}$, 53.73; H, 5.41; N, 12.53. Found: C, 53.53; H, 5.31; N, 12.50\%.

Diethyl (5-methylisoxazole-3-ylamino)(1H-indol-3-yl)methyl phosphonate (4e). Yield 80\% mp 73-74 ${ }^{\circ}$; IR (KBr) cm ${ }^{-1} ; 3220(\mathrm{P}-\mathrm{NH}), 1230(\mathrm{P}=\mathrm{O}), 755(\mathrm{P}-\mathrm{CH}) ;{ }^{1} \mathrm{H}-\mathrm{NMR}$ (DMSO- $\left.d_{6}\right) \delta$ 6.80-7.94 (m, 6H, Ar-H), 10.12 (s, 1H, Ar-NH), 5.71 (s, 1H, Aliph-NH), 4.75-4.93 (m, 1H,P$\mathrm{CH})$; $3.71-3.89$ (m, 4H, P-O- $\left.\underline{\mathrm{CH}}_{2}-\mathrm{CH}_{3}\right), 1.49-1.52$ (t, 6H, P-O-CH $\left.2-\underline{\mathrm{H}}_{3}\right), 2.15(\mathrm{~s}, 3 \mathrm{H}, \mathrm{Ar}-\mathrm{CH} 3) ;{ }^{31} \mathrm{P}$ NMR (DMSO- $\left.d_{6}\right) ; \delta$ 28.62. Anal. Calcd. for $\mathrm{C}_{17} \mathrm{H}_{22} \mathrm{~N}_{3} \mathrm{O}_{4} \mathrm{P}: \mathrm{C}, 56.03 ; \mathrm{H}, 6.36 ; \mathrm{N}, 11.53$. Found: C, 55.95; H, 6.25; N, 11.49\%.

Diphenyl (5-methylisoxazole-3-ylamino)(1H-indol-3-yl)methyl phosphonate (4f). Yield 75\% mp 73-74 ${ }^{\circ}$; IR (KBr) cm ${ }^{-1} ; 3386(\mathrm{P}-\mathrm{NH}), 1235(\mathrm{P}=\mathrm{O}), 766(\mathrm{P}-\mathrm{CH}) ;{ }^{1} \mathrm{H}-\mathrm{NMR}\left(\mathrm{DMSO}-d_{6}\right) \delta$ 
6.60-7.94 (m, 16H, Ar-H), 9.90 (s, 1H, Ar-NH), 5.81 (s, 1H, Aliph-NH), 4.85-4.89 (m, 1H, P$\mathrm{CH}) ; 2.50\left(\mathrm{~m}, 3 \mathrm{H}, \mathrm{Ar}-\mathrm{CH}_{3}\right) .{ }^{31} \mathrm{P}$ NMR (DMSO-d $)$; $\delta$ 26.76. Anal. Calcd. for $\mathrm{C}_{25} \mathrm{H}_{22} \mathrm{~N}_{3} \mathrm{O}_{4} \mathrm{P}: \mathrm{C}$, 65.22; H, 6.43; N, 9.12. Found: C, 65.10; H, 6.38; N, 9.08\%.

Dimethyl ((ethoxycarbonyl)(phenyl)methylamino)(1H-indol-3-yl)methyl phosphonate (4g). Yield (65\%) mp 145-146 C; IR (KBr) cm ${ }^{-1}$; 3397 (P-NH), $1238(\mathrm{P}=\mathrm{O}), 758(\mathrm{P}-\mathrm{CH})$; ${ }^{1} \mathrm{H}-\mathrm{NMR}$ $\left(\mathrm{DMSO}-d_{6}\right) \delta 6.72(\mathrm{~m}, 10 \mathrm{H}, \mathrm{Ar}-\mathrm{H}), 9.97$ (s, 1H, Ar-NH), 5.14 (s, 1H, Aliph-NH), 4.86-4.90 (m, $1 \mathrm{H}, \mathrm{P}-\mathrm{CH}) ; 3.31-3.39\left(\mathrm{~d},{ }^{3} J_{\mathrm{P}-\mathrm{H}}=10.1 \mathrm{~Hz}, \mathrm{P}-\mathrm{OCH}_{3}\right) ; 3.55-3.65\left(\mathrm{~d},{ }^{3} J_{\mathrm{P}-\mathrm{H}}=11.3 \mathrm{~Hz}, \mathrm{P}-\mathrm{OCH}_{3}\right)$; 3.69-3.74 (m, 2H, $\left.\underline{\mathrm{OCH}}_{2}-\mathrm{CH}_{3}\right), 1.34-1.36\left(\mathrm{t}, 3 \mathrm{H}, \mathrm{O}-\mathrm{CH}_{2}-\underline{\mathrm{CH}}_{3}\right) .{ }^{31} \mathrm{P}$ NMR (DMSO-d 6 ); $\delta: 31.30$. Anal. Calcd. for $\mathrm{C}_{21} \mathrm{H}_{25} \mathrm{~N}_{2} \mathrm{O}_{5} \mathrm{P}: \mathrm{C}, 60.58 ; \mathrm{H}, 6.05 ; \mathrm{N}, 6.72$. Found: C, 60.38; H, 5.90; N, 6.65\%.

Diethyl ((ethoxycarbonyl)(phenyl)methylamino)(1H-indol-3-yl)methyl phosphonate (4h). Yield (65\%) mp 145-146 ${ }^{\circ}$; IR (KBr) cm ${ }^{-1}$; $3397(\mathrm{P}-\mathrm{NH}), 1238(\mathrm{P}=\mathrm{O}), 758(\mathrm{P}-\mathrm{CH})$; ${ }^{1} \mathrm{H}-\mathrm{NMR}$ $\left(\mathrm{DMSO}-d_{6}\right) \delta$ 6.72-7.86 (m, 10H, Ar-H), 9.97 (s, 1H, Ar-NH), 5.14 (s, 1H, Aliph-NH), 4.70-4.85 (m, 1H,P-CH); 3.89-3.90 (m, 4H, P-O-C $\left.\underline{H}_{2}-\mathrm{CH}_{3}\right), 1.39-1.45$ (m,6H, P-O-CH$\left.-\mathrm{CH}_{3}\right), 3.29-3.31$ $\left(\mathrm{m}, 2 \mathrm{H}\right.$, amino-OC$\left.\underline{H}_{2}\right), 1.34-1.36\left(\mathrm{t}, 3 \mathrm{H}-\mathrm{O}-\mathrm{CH}_{2}-\mathrm{CH}_{3}\right) .{ }^{31} \mathrm{P}$ NMR (DMSO- $\left.d_{6}\right) \delta 31.30$. Anal. Calcd. for $\mathrm{C}_{21} \mathrm{H}_{25} \mathrm{~N}_{2} \mathrm{O}_{5} \mathrm{P}: \mathrm{C}, 60.58 ; \mathrm{H}, 6.05 ; \mathrm{N}, 6.72$. Found: C, 60.48; H, 5.92; N, 6.61\%.

Diphenyl ((ethoxycarbonyl)(phenyl)methylamino)(1H-indol-3-yl)methyl phosphonate (4i). Yield (65\%) mp 168-169 ${ }^{\circ}$; IR (KBr) cm ${ }^{-1}$; 3360 (P-NH), $1209(\mathrm{P}=\mathrm{O}), 786(\mathrm{P}-\mathrm{CH}) ;{ }^{1} \mathrm{H}$ NMR $\left(\mathrm{DMSO}-d_{6}\right) \delta: 6.80-7.96(\mathrm{~m}, 16 \mathrm{H}, \mathrm{Ar}-\mathrm{H}), 9.91$ (s, 1H, Ar-NH), 5.38 (s, 1H, Aliph-NH), 4.76$4.80(\mathrm{~m}, 1 \mathrm{H}, \mathrm{P}-\mathrm{CH}), 3.20-3.29\left(\mathrm{~m}, 2 \mathrm{H}, \mathrm{OCH}_{2}\right), 1.28-1.37\left(\mathrm{t}, 3 \mathrm{H}, \mathrm{O}-\mathrm{CH}_{2} \mathrm{CH}_{3}\right) .{ }^{31} \mathrm{P}$ NMR (DMSO- $d_{6}$ ) $\delta$ 20.18. Anal. Calcd. for $\mathrm{C}_{31} \mathrm{H}_{29} \mathrm{~N}_{2} \mathrm{O}_{5} \mathrm{P}: \mathrm{C}, 68.88 ; \mathrm{H}, 5.40 ; \mathrm{N}, 5.18$. Found: C, 68.64; H, 5.26; N, 5.10\%.

Dimethyl (cyclohexylamino)(1H-indol-3-yl)methyl phosphonate (4j). Yield (85\%) mp 160$161^{\circ} \mathrm{C}$; IR (KBr) cm ${ }^{-1} 3359$ (P-NH), $1208(\mathrm{P}=\mathrm{O}), 773(\mathrm{P}-\mathrm{CH}) .{ }^{1} \mathrm{H}-\mathrm{NMR}$ (DMSO-d $)$ ) $\delta$ 7.20-7.68 (m, 6H, Ar-H), 9.86 (s, 1H, Ar-NH), 4.8 (s, 1H Aliph-NH), 4.79-4.84 (m, 1H, P-CH), 3.42-3.49 $\left(\mathrm{d},{ }^{3} J_{\mathrm{P}-\mathrm{H}}=11.4 \mathrm{~Hz}, \mathrm{P}-\mathrm{OCH}_{3}\right), 3.55-3.67\left(\mathrm{~d},{ }^{3} J_{\mathrm{P}-\mathrm{H}}=9.80 \mathrm{~Hz}, \mathrm{P}-\mathrm{OCH}_{3}\right) ; 1.98-2.50(\mathrm{~m} 11 \mathrm{H}$, cyclohexyl-H). ${ }^{13} \mathrm{C}$ NMR (DMSO- $\left.d_{6}\right) \delta 122.2$ (C-2), 110.2 (C-3), 121.0 (C-4), 120.2 (C-5), 118.2 (C-6), 113.9 (C-7), 131.2 (C-8), 137.8 (C-9), 34.8 (C-1'), 22.0 (C-2'), 28.1 (C-3'), 22.3 (C4'), 34.0 (C-5"), $52.0\left(\mathrm{C}-6\right.$ '), $54.9(\mathrm{P}-\mathrm{CH}), 53.8\left(\mathrm{O}-\mathrm{CH}_{3} \mathrm{~d}, J=6.4 \mathrm{~Hz}\right) .{ }^{31} \mathrm{P}$ NMR (DMSO- $\left.d_{6}\right) \delta$ 31.80. Anal. Calcd. for $\mathrm{C}_{17} \mathrm{H}_{25} \mathrm{~N}_{2} \mathrm{O}_{3} \mathrm{P}: \mathrm{C}, 60.74 ; \mathrm{H}, 7.49 ; \mathrm{N}, 8.32$. Found: C, 60.56; H, 7.38; N, $8.36 \%$.

Diethyl (cyclohexylamino)(1H-indol-3-yl)methyl phosphonate (4k). Yield (80\%) mp 170$171^{\circ} \mathrm{C}$; IR (KBr) cm ${ }^{-1} ; 3389(\mathrm{P}-\mathrm{NH}), 1236(\mathrm{P}=\mathrm{O}), 770(\mathrm{P}-\mathrm{CH}) ;{ }^{1} \mathrm{H}-\mathrm{NMR}\left(\mathrm{DMSO}-d_{6}\right) \delta$ 7.09-7.78 (m, 6H, Ar-H), 9.86 (s, 1H, Ar-NH), 4.96 (s, 1H, Aliph-NH), 4.77-4.81 (m, 1H, P-CH), 3.753.78 (m, 4H, P-O- $\left.\underline{\mathrm{CH}}_{2}-\mathrm{CH}_{3}\right), 1.35-1.37$ (m, 6H, P-O-CH$\left.{ }_{2}-\underline{\mathrm{C}}_{3}\right)$, 1.97-2.6 (m, 11H, cyclohexylH). ${ }^{31} \mathrm{P}$ NMR (DMSO- $d_{6}$ ); $\delta$ 29.27. GC-MS m/z (\%): 365.0 (45), 331 (8), 266.0 (100), 237.9 (5), 227.0 (60), 156.0 (18), 145.0 (5), 130.0 (11). Anal. Calcd. for $\mathrm{C}_{19} \mathrm{H}_{29} \mathrm{~N}_{2} \mathrm{O}_{3} \mathrm{P}: \mathrm{C}, 62.62 ; \mathrm{H}, 8.01$; N, 7.68. Found: C, 62.41; H, 7.90; N, 7.56\%.

Diphenyl (cyclohexylamino)(1H-indol-3-yl)methyl phosphonate (4l). Yield (85\%) mp 115$116^{\circ} \mathrm{C}$; IR ( $\left.\mathrm{KBr}\right) \mathrm{cm}^{-1} ; 3378(\mathrm{P}-\mathrm{NH}), 1228(\mathrm{P}=\mathrm{O}), 775(\mathrm{P}-\mathrm{CH}) ;{ }^{1} \mathrm{H}-\mathrm{NMR}\left(\mathrm{DMSO}-d_{6}\right) \delta$ 6.90-7.68 (m, 16H, Ar-H), 9.76 (s, 1H, Ar-NH), 4.91 (s, 1H, Aliph-NH), 4.78-4.82 (m,1H, P-CH), 1.86- 
$2.56\left(\mathrm{~m}, 11 \mathrm{H}\right.$, cyclohexyl-H). ${ }^{13} \mathrm{C}$ NMR (DMSO- $\left.d_{6}\right) \delta 122.6$ (C-2), $111.0(\mathrm{C}-3), 121.5(\mathrm{C}-4)$, 120.2 (C-5), 119.0 (C-6), 114.0 (C-7), 131.8 (C-8), 138.5 (C-9), 35.2 (C-1'), 21.5 (C-2'), 28.2 (C3'), 22.4 (C-4'), 35.0 (C-5'), 51.5 (C-6'), 136.5(C-1"), 130.6(C-2"), 129.6(C-3"), 132.5(C-4"), 128.9(C-5"), $130.2(\mathrm{C}-6 "), 54.5(\mathrm{P}-\mathrm{CH}) ;{ }^{31} \mathrm{P}$ NMR (DMSO- $\left.d_{6}\right) \delta$ 14.67. Anal. Calcd. for $\mathrm{C}_{27} \mathrm{H}_{29} \mathrm{~N}_{2} \mathrm{O}_{3} \mathrm{P}$ : C, 70.42; H, 6.34; N, 6.08. Found: C, 70.26; H, 6.17; N, 6.00\%.

\section{Conclusions}

In our current search, we have reported the synthesis of $\alpha$-aminophosphonates in one-pot synthesis from aldehyde, amines and dialkyl, diaryl phosphite using tetramethylguanidine as catalyst which includes one more method for its synthesis to the existing literature.

\section{Acknowledgements}

The authors thank C. Naga Raju, Dept. of Chemistry, S.V. University, Tirupati for helpful discussions. The authors thank Department of Atomic Energy (BRNS), Mumbai, India for providing financial assistance.

\section{References}

1. Kaboudin, B.; Nazari, R. Tetrahedron Lett. 2001, 42, 8211.

2. Matveeva, E. D.; Podrugina, T. A.; Tishkovskaya, E. V.; Tomilova, L. G.; Zefirov, N. S. Synlett 2003, 2321.

3. Pavlov, V. Ya.; Kabachinik, M. M.; Zobnina, E. V.; Ponomarev, G. V.; Beletskaya, I. P. Synlett 2003, 2193.

4. Sikorski, J. A.; Miller, M. J.; Braccolino, D. S.; Cleary, D. G.; Corey, S. D.; Ream, J. E.; Schnur, D.; Shah, A.; Walker, M. C. Phosphorus, Sulfur, Silicon 1993, 76, 375.

5. Stowasser, B.; Budt, K. H.; Jain-Qi, L.; Peyman, A.; Ruppert, D. Tetrahedron Lett. 1992, 33, 6625.

6. Patel, D. V.; Reilly-Gauvin, K.; Ryono, D. E. Tetrahedron Lett. 1990, 31, 5587.

7. (a) Bruke, T. R., Jr.; Brachi, J. J., Jr.; George, C.; Wolf, G.; Shoelson, S. E.; Yan, X. J. Med. Chem. 1995, 38, 1386. (b) Bruke, T. R., Jr.; Kole, H. K.; Roller, P. P. Biochem. Biophys. Res. Commun. 1994, 204, 129.

8. Peyman, A.; Budt, K. H.; Paning, J. S.; Stowasser, B.; Ruppert, D. Tetrahedron Lett. 1992, $33,4549$.

9. Atherton, F. R.; Hassall, C. H.; Lambert, R. W. J. Med. Chem. 1986, 29, 29. 
10. Kafarski, P.; Lejack, B.; Mastalerz, P. Beitr. Wirk. Forsh. 1985, H25; Chem. Abstr. 1985, 103, 174532.

11. (a) Maier, L.; Lea, P. J. Phosphorus Sulfur Silicon 1983, 17, 1. (b) Giannousis, P. P.; Bartlett, P. A. J. Med. Chem. 1987, 30, 1603. (c) Gancarz, R.; Wieczorek, J. S. Synthesis, 1977, 625. (d) Baylis, E. K.; Campbell, C. D.; Dingwall, J. G. J. Chem. Soc., Perkin Trans. 1 1984, 2845.

12. Fields, S. C. Tetrahedron 1999, 55, 12237.

13. Kleszczynska, H.; Sarapuk, J. Cell. Mol. Biol. Lett., 2001, 6, 83.

14. (a) Kabachinic, M. J.; Medved, T. Izv. Akad. Nauk. SSSR 1953, 1126. (b) Kabachinic, M. J.; Medved, T. Izv. Akad. Nauk. SSSR 1954, 1024.

15. Fields, E. K. J. Am. Chem. Soc. 1952, 74, 1528.

16. Pollini, G. P.; Barco, A.; Degiuli, G. Synthesis 1924, 44.

17. Hewson, A. T., Macpherson, D. T. Tetrahedron Lett. 1983, 24, 647.

18. Silverstein, R. M.; Webster. F. X. Spectrometric Identification of Organic Compounds, $6^{\text {th }}$ Edn.:Wiley: New York, 1998.

19. Tongcharoensirikul, P.; Suarez, A.I.; Voelker, T.; Thomson, C. M. J. Org. Chem. 2004, 69, 2322.

20. Heydari, A.; Zarei, M.; Alijaninzadeh, R.; Tavakol, H. Tetrahedron Lett. 2001, 42, 3629.

21. Qian, C.; Huang, T. J. Org. Chem., 1998, 63, 4125.

22. Semenzin, D.; Moghadam, G. E.; Albiouy, D.; Diallo O.; Koenig, M. J. Org. Chem., 1997, $62,2414$.

23. Quin, L. D.; Verkade, J. G. Phosphorus-31 NMR Spectral Properties in Compound Characterization and Structural Analysis; VCH:New York, 1994

24. Vincent, J. C.; Vincent, H. W. Proc. Soc. Expt. Biol. Med. 1944, 55, 162.

25. Benson, H. J. Microbiological Applications, $5^{\text {th }}$ Edn., W.C. Brown Publications: Boston, 1990. 